\title{
Virtualization for Load Balancing on IEEE 802.11 Networks
}

\author{
Tibério M. de Oliveira ${ }^{1}$, Marcel W. R. da Silva, Kleber V. Cardoso ${ }^{2}$, and José \\ Ferreira de Rezende ${ }^{1}$ \\ 1 Universidade Federal do Rio de Janeiro - Rio de Janeiro, RJ, Brazil \\ tiberio, marcel, rezende@gta.ufrj.br, \\ 2 Universidade Federal de Goiás - Goiânia, GO, Brazil \\ kleber@inf.ufg.br
}

\begin{abstract}
In IEEE 802.11 networks composed by multiple APs, before a station can access the network it needs to make a decision about which AP to associate with. Usually, legacy 802.11 stations use no more than the signal strength of the frames received from each AP to support their decision. This can lead to an unbalanced distribution of stations among the APs, causing performance and unfairness problems. This work proposes a new approach that combines the number of associated stations and the current load of each AP plus the virtualization of client wireless interfaces. In this approach, stations permanently switch of association among the APs, staying on each AP for a time that takes into account the number of associated stations and the current load in each AP. Simulation results confirm the improvement obtained in the load balancing and fairness on network capacity allocation, while keeping the maximum network utilization.
\end{abstract}

Key words: wireless networks, scheduling, 802.11, association

\section{Introduction}

Nowadays, there is a large number of IEEE 802.11 access points (APs) available in both private and public access networks. Before a client station (STA) can have access to the data transmission service provided by such networks, it has to follow procedures of association and authentication with one of the APs in its transmission range. To do this, the STA firstly detects APs in its vicinity by scanning wireless channels and collecting responses (probe responses and/or beacon frames) from them. Then, the STA authenticates and associates with the AP from which it received frames with the highest RSSI (Received Signal Strength Indicator).

As presented in [1], this association metric does not ensure efficiency in the resources usage and may lead to a poor performance due to the unbalanced number of associated stations among the APs. Alternative approaches have been proposed [1, 2, 3], which perform a load balancing among APs by including load conditions in the frames in order to allow the STA to select the least loaded AP. 
However, the advertised load only considers the number of stations associated to the AP. Thus, these works do not evaluate the effective amount of traffic generated by the stations.

Our proposal takes into consideration the effective throughput achieved by the stations and uses wireless network interface virtualization [4] to perform load balancing. The virtualization allows that one single physical interface is used to offer connectivity to more than one AP [5]. Additionally, it can schedule virtual interfaces to balance the load in both stations and APs. Through a simulation study, we show that this scheme outperforms the standard RSSI-based association and other approach for load balancing known as DLBA (Dynamic Load Balancing Algorithm) [3].

The rest of this paper is organized as follows. In Section 2, we briefly present some important related works. Section 3 exposes some important concepts about load balancing and IEEE 802.11 network virtualization. Section 4 presents our proposal of load balancing through network virtualization. In Section 5, we show the results in comparison with some other approaches. Finally, in Section 6, we present our conclusion.

\section{Related Work}

Recently, virtualization has become an important tool in several areas, such as operating systems [6], faults detection and diagnosis [7]. In wireless networks, it has been applied in the handover process $[8,9]$ and network/interfaces virtualization $[10,11,4,5]$.

The IEEE 802.11 physical interface virtualization allows an STA to associate with multiple APs simultaneously. This capability can be used with the purpose of allowing the concurrent access to multiple networks or virtually increasing the connectivity in a unique infrastructure network [5]. In this last scenario, it is also required that STAs constantly change their association among the APs in order to announce their presence. Currently, the traditional association approach takes into account only the RSSI measured by STAs from multiple APs. The main drawback of this approach is that it can lead to an unbalanced distribution of STAs among the APs, which can drastically reduce network performance [3, 1 , $9,12]$.

To circumvent this problem, some metrics for AP association that define a relation between RSSI and the amount of associated stations to an AP were proposed $[3,1,2]$. In this work, we propose a load balancing mechanism based on IEEE 802.11 physical interface virtualization, which uses a metric derived from the amount of associated STAs and channel load to determine how long an STA will stay associated with each AP. This proposal uses a virtualization scheme called Frequency Hopping $(\mathrm{FH})$ and prioritizes the AP that has the lowest channel occupation. 


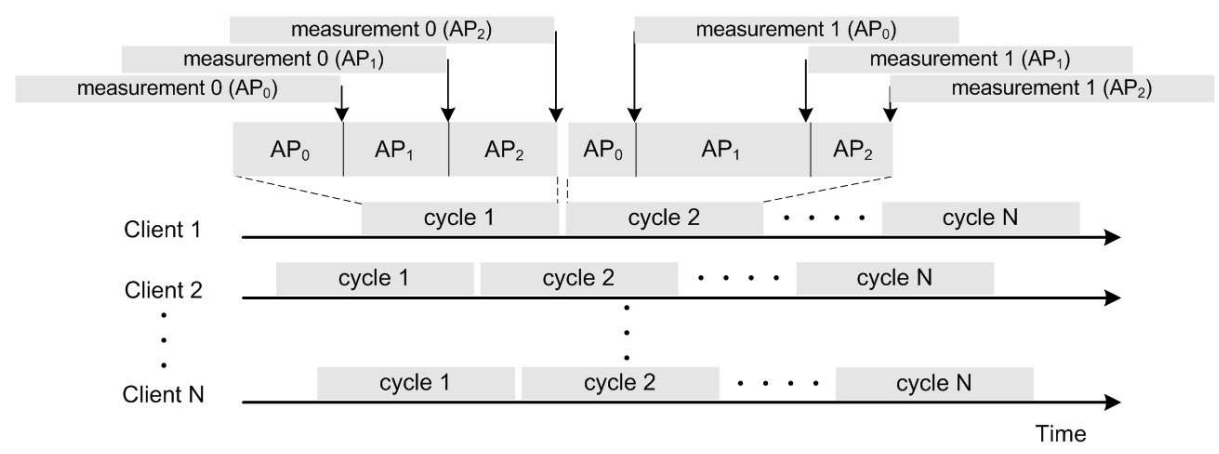

Fig. 1. Our load balance approach by AP hopping virtualization.

\section{Background}

\subsection{IEEE 802.11 Network Virtualization}

The main virtualization focus is to allow a single substrate to execute multiple virtual experiments. The wireless medium can be virtualized by different techniques as presented in [4]. The most common are: FDMA, TDMA, Combined TDMA and FDMA, CDMA, SDMA, and Frequency Hoping (FH).

The FH scheme is a dynamic version of the Combined TDMA and FDMA scheme. It allows experiment partitioning by allocating a unique sequence of frequency and time slots for each virtual experiment. However, differently from the Combined TDMA and FDMA scheme, it allows that the same experiment uses different sequences. It is the most flexible and complete scheme to be used with IEEE 802.11 networks.

Our mechanism uses the FH scheme combined with an algorithm to choose the sequences, i.e. channels and time slot sizes to be used by each STA. The algorithm employs a metric based on media occupation and the amount of associated STAs to each AP. Both IEEE 802.11k and 802.11r standards are used to implement our proposal. The proposed metric requires some data collected by the IEEE $802.11 \mathrm{k}$, and the wireless interface virtualization makes use of the IEEE $802.11 \mathrm{r}$ for fast handover.

\subsection{IEEE 802.11k and IEEE 802.11r standards}

The IEEE $802.11 \mathrm{k}$ team (Radio Resource Measurement) is developing a standard that aims to increase the physical layer and medium access availability. For this purpose, it defines a sequence of measurements requests and reports about radio and network information that can be used by upper layers in different ways. It presents low overhead of control messages and low processing requirements, and provides enough accurate measurements for our proposal.

The radio measurements in wireless networks help applications to adapt automatically to the dynamic medium conditions, facilitating the management 
and maintenance of a WLAN. The standard specifies a generic pair of Radio Measurement Request and Radio Measurement Report frames, which can be specialized to acquire measurements such as channel load, STA statistics, number of neighbors, etc.

When an STA switches between APs in a handover process, it starts a reassociation with the new AP. During this process, the IEEE 802.11r implemented in the APs takes care of changing forwarding tables of L2 devices by issuing gratuitous ARP messages on the distribution system. It also allows the involved APs to exchange authentication information, which drastically reduces the time spent in the handover process.

\section{Load Balancing by using Virtual Interfaces}

In general, a suitable load balancing provides an efficient use of resources, which in most cases improves fairness in their allocation. In other words, while properly distributing STAs across APs, the aggregate network throughput is increased and the fairness in the network capacity allocation is improved. The DLBA association algorithm balances the number of STAs associated to each AP by using the RSSI. However, since STAs may present different traffic profiles, this is not enough to proper balance the network load and, as a consequence, it is not suitable for providing throughput or delay fairness.

In this context, we propose a new load balancing mechanism based on the time slot scheduling of a frequency hopping virtualization technique. This mechanism is distributed and provides effective fairness in the network resources allocation. Also, it is standard-compliant and completely implemented in the STAs, so it does not require modifications in the APs.

In the proposed mechanism, the STAs manage associations with multiple APs using one virtual wireless interface for each AP. An STA stays associated to each AP for a time interval that is dynamically adjusted by the scheduling algorithm, which takes into account measurements of channel occupation and number of STAs associated to each AP. This time interval is always a fraction of a scheduling cycle and is called the active time of a virtual interface.

Fig. 1 illustrates the scheduling process in which an STA periodically chooses a different AP to associate with. When the APs in the STA's coverage area operate in different channels ${ }^{1}$, the scheduling process becomes a client-based $\mathrm{FH}$ virtualization scheme.

Just before the end of an active time, the STA collects from the current AP information about channel occupation and number of associated STAs during this active time. In the end of every scheduling cycle, the STA uses the collected measurements to compute fractions (or weights) for the calculation of the active time of each virtual interface in the next scheduling cycle. The measurements are performed and made available by the APs using IEEE $802.11 \mathrm{k}$.

\footnotetext{
${ }^{1}$ Our mechanism does not depend on an efficient channel allocation, but this is a common assumption in infrastructure networks with centralized control.
} 
In Fig. 1, STA 1 has three virtual interfaces since it is in the coverage area of three $\mathrm{APs}^{2}$. To avoid synchronization of active times of different STAs on the same AP, every active time is randomly varied by $10 \%$ of its current computed value.

In order to keep the unawareness of the APs concerning the load balancing, our mechanism employs the IEEE 802.11r protocol to implement a fast handover process with authentication. It allows an uninterrupted forwarding of downlink traffic during virtual interface switching. As illustrated in Fig. 2, when an STA switches to a new $\operatorname{AP}\left(A P_{1}\right)$, it performs a fast authentication before reassociating, and then initiates an IEEE 802.11X authentication. After that, the IEEE 802.11r 4-way handshake is performed between STA and new AP. If this operation is successful, the new AP updates the L2 forwarding tables of the distribution system equipments. The whole process of migrating from one AP to another takes a non-negligible time. In IEEE $802.11 \mathrm{r}$, this time is around 40 and $50 \mathrm{~ms}$ (excluding the scanning time) [13].

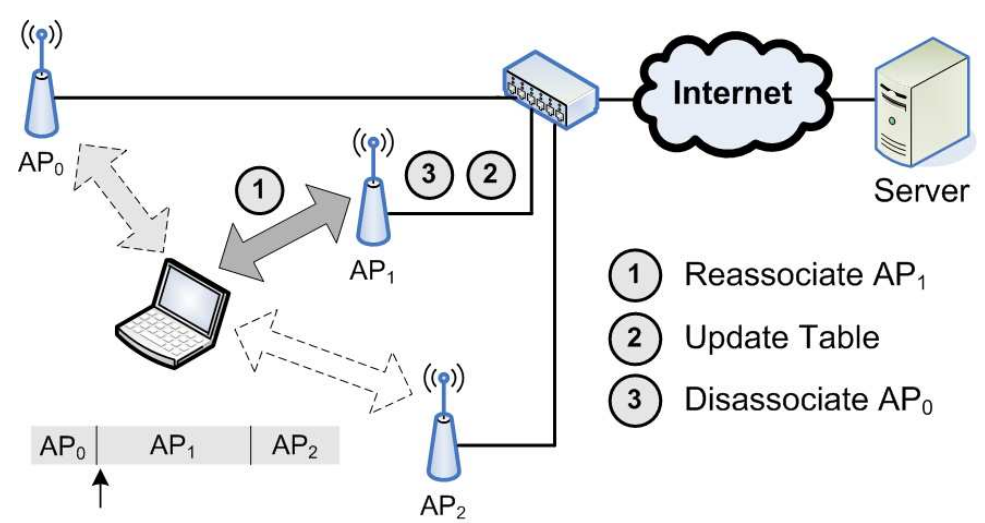

Fig. 2. Fast handover during virtual interface switching.

\subsection{Active Time Computation Algorithm}

The performance of the proposed load balancing mechanism is closely tied to the active time duration of each virtual interface. After each cycle, these durations are recomputed to be used in the next cycle. These updates are based on channel load and the number of associated STAs per AP. Initially, the mechanism computes an estimate of the idle time for each STA associated to $A P(i)$, as described by Equation 1:

\footnotetext{
2 This number may vary from cycle to cycle.
} 


$$
\left\{\begin{array}{l}
s \in A P(i), \quad \overline{I_{(i)}}(s)=\frac{1-C h_{\text {Load }}(i)}{N S t(i)}, \\
s \notin A P(i), \quad \overline{I_{(i)}}(s)=\frac{1-C h_{\text {Load }}(i)}{N S t(i)+1}
\end{array}\right.
$$

where $\overline{I_{(i)}}(s)$ represents the average amount of time the $A P(i)$ stayed idle per associated STA, $C h_{\text {Load }}(i)$ is the last channel load reported by the $A P(i)$, and $N S t(i)$ is the number of STAs associated to $A P(i)$. When the STA $s$ is not yet associated to $A P(i)$, the equation includes the STA $s$ as if it was already associated to $A P(i)$ in order to artificially account for its contribution to the load.

Finally, to determine the time slots duration, weights are assigned to each one. The sum of all time slots is equal to the duration of a scheduling cycle, and the number of time slots is equal to the number of $\mathrm{APs}\left(N_{A P s}\right)$. Hence, the weights $\left(W_{(i)}(s)\right)$ computed by an STA $s$ are normalized by the cycle duration to obtain a percentage of the cycle. This computation is described by Equation 2:

$$
W_{(i)}(s)=\frac{\overline{I_{(i)}}(s)}{\sum_{k=1}^{N_{A P s}} \overline{I_{(k)}}(s)} .
$$

The adaptive active time duration allows our mechanism to dynamically track the network load. For example, an STA allocates larger time slots to APs that have a lower amount of load per associated STA. Therefore, the proposed virtualization mechanism provides a dynamic load balancing across the APs while performing a fair resource sharing between STAs.

\section{Results}

The ns-2 simulator with a set of modifications was used to evaluate the proposed load balancing mechanism. At the network and MAC layers, we added interface queues for the traffic of each virtual interface. Modifications were also needed to allow $802.11 \mathrm{k}$ measurements of the number of associated STAs, channel occupation and RSSI. Moreover, we implemented the proposed load balancing mechanism and other association mechanisms that served for performance comparisons.

Before evaluating the proposed mechanism in commonly used IEEE 802.11 infrastructure scenarios, we performed some experiments to assess the overhead imposed by the virtualization. The delay incurred when an STA virtualizes to a new AP, called here of virtualization delay, occurs because of channel switching, reassociation, authentication and L2 forwarding tables update. During this time, all in transit packets can be lost, degrading flows performance. To do this evaluation, we run simulations with only one virtualized STA in the range of three APs interconnected by a unique L2 switch. This STA performs an FTP download during all the experiment. Fig. 3 shows the FTP flow average throughput normalized 


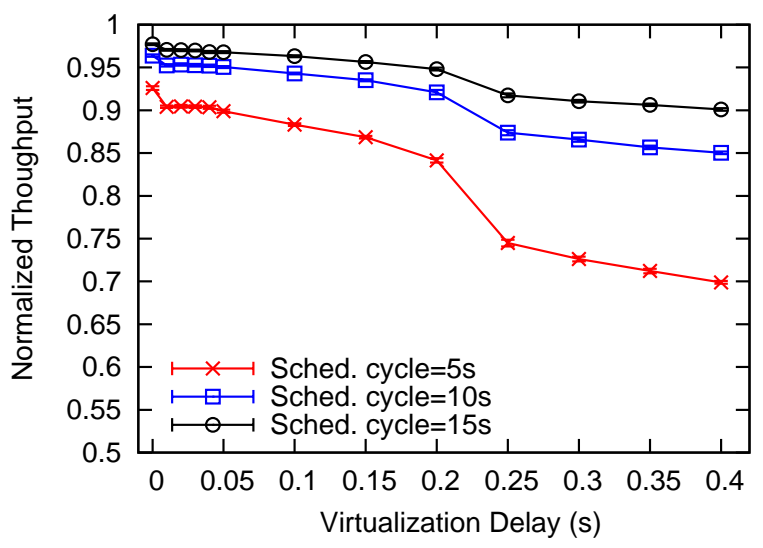

Fig. 3. Virtualization overhead.

in respect to the throughput obtained by simulations when no virtualization is used. This normalization helps to analyze the performance degradation due to the delay explained above. The normalized average throughput is plotted as a function of the virtualization delay for different scheduling cycle durations. According to [9], typical values for the virtualization delay are not larger than 40 $m s$. Results show that the throughput decreases by at most $10 \%$ in the range of 0 and $40 \mathrm{~ms}$. When the duration of the schedule cycle decreases, the flow throughput also decreases since the STA virtualizes more often. When a channel scanning overhead, which has a typical value of $350 \mathrm{~ms}$, is added to the virtualization delay, the performance degradation is severe. However, our mechanism does not require channel scanning since each STA already knows which APs it is virtualizing with.

To evaluate the performance of the proposed mechanism the next simulations involve an infrastructure network composed by three APs disposed at the center of a square area, respecting a minimum distance of 120 meters among them. Each AP is configured to use one of the non-overlapping channels (1, 6 and 11).

Two classes of STAs were positioned in the communication range of these three APs: legacy and special STAs. Legacy STAs receive download Web traffic and use a traditional association method. Special STAs receive download FTP traffic during all simulation. We performed simulations in different scenarios where the special STAs use one of the following association methods:

- Legacy: Traditional association method, the same used by the legacy stations [14];

- DLBA: The DLBA association mechanism [3];

- Static Virtualization: The proposed association mechanism with static time slot duration;

- Dynamic Virtualization: The proposed association mechanism with dynamic time slot durations. 


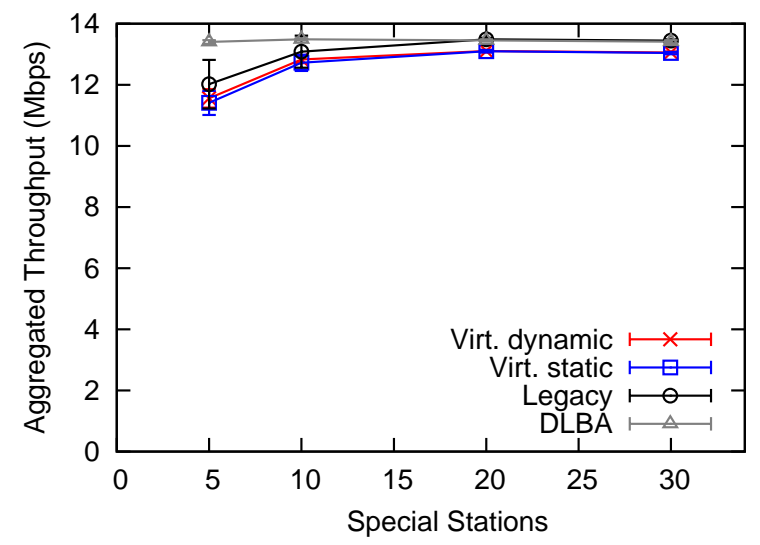

(a) Without legacy stations, only special stations.

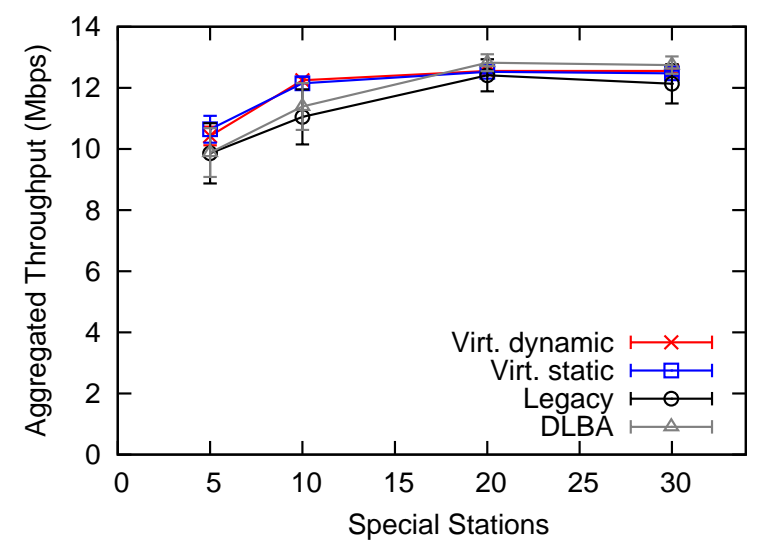

(b) 30 legacy stations with Web traffic.

Fig. 4. Aggregated throughput of the FTP flows generated to special STAs as a function of the number of special STAs.

Each simulation run lasted for $200 \mathrm{~s}$ and 30 different STAs positioning scenarios were generated for each configuration set. The results presented are the mean of obtained values for the 30 scenarios with confidence interval bounds at a confidence level of $95 \%$.

Figure 4 presents the aggregate throughput of the special STAs in two scenarios, without and with 30 legacy STAs. The aggregate throughput is a metric given by the sum of all FTP flows throughput. The results show that all association methods have similar performance. As the TCP used by FTP applications always try to grab all available channel capacity, the aggregate throughput reaches the maximum network capacity in most scenarios. Thus, the virtualization mechanism presents aggregate performance similar to the other evaluated schemes even performing load balancing of STAs between the APs. Despite vir- 


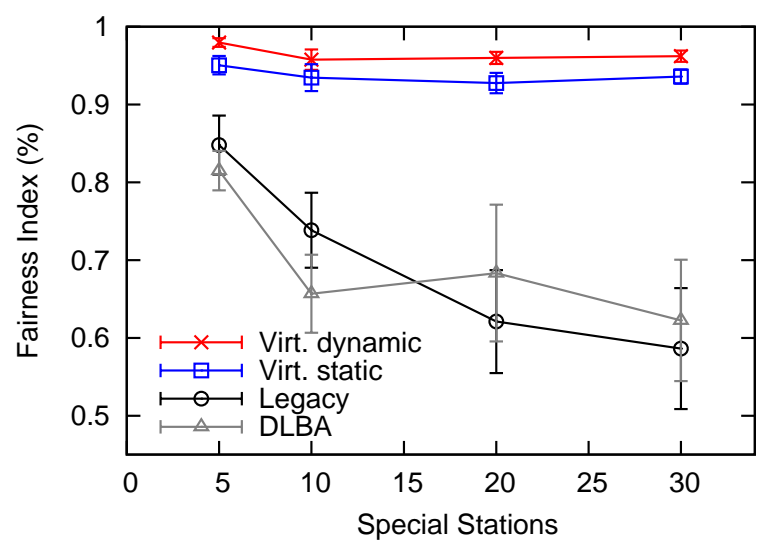

(a) Only special STAs.

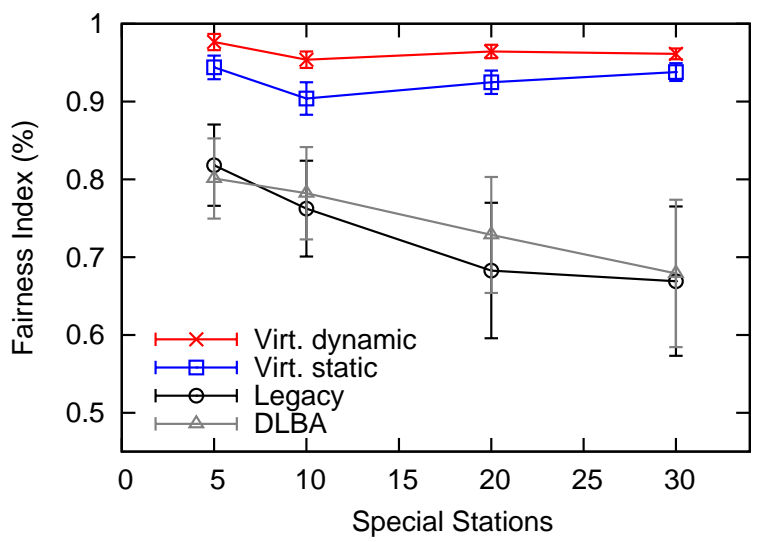

(b) 30 legacy STAs with Web traffic.

Fig. 5. Fairness index.

tualized stations being penalized by the cost of switching channels, this does not prevent these stations to attain a performance close to the maximum.

Figure 5 shows the fairness index, defined in [15], calculated using special STA throughputs. It represents how fair the network capacity is distributed among the STAs. The closer this index is to 1 , the fairer is the distribution. According to Fig. 5, the fairness index of virtualized stations is the best among all association algorithms. This demonstrates that our proposal provides a better load balancing, ensuring a fairer share of network resources. The difference between static and dynamic virtualized stations is related to the fact that the static algorithm keeps bad associations for a longer time. DLBA and Legacy association algorithms present smaller fairness indexes and larger variance.

Other important metric is presented in Fig. 6. This figure shows the web page average latency obtained by legacy STAs when the amount of special STAs 


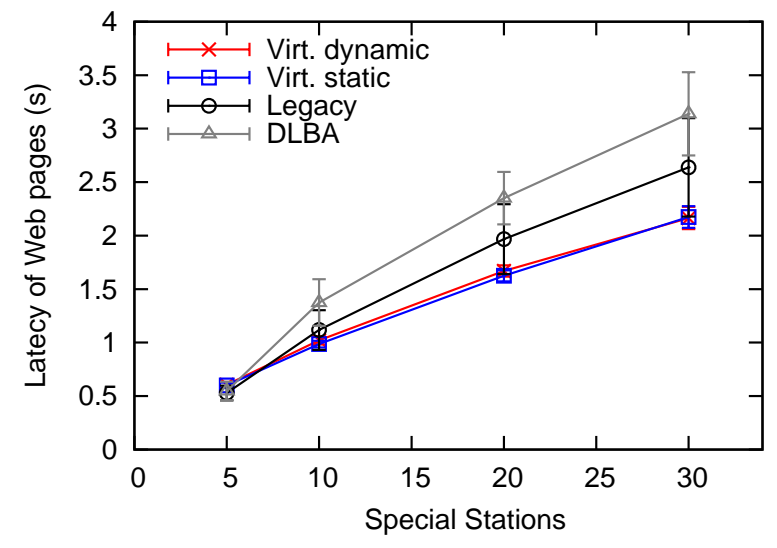

Fig. 6. Average of web pages latency for legacy STAs.

increases. This metric allows evaluating how friendly the association algorithm is. Once again, DLBA and Legacy STAs present a lower performance with a significant increase in the average latency to visualize web pages. The virtualized methods have a low impact in the average latency of web pages as the amount of special STAs increases.

\section{Conclusions}

This work has presented a new mechanism that uses IEEE 802.11 network interfaces virtualization as a way to perform load balancing. This proposal has been evaluated and compared through simulations to a traditional approach and another load balancing proposal that takes into account only the RSSI. Simulation results show that the virtualization overhead is negligible and the full aggregate capacity of the network can be achieved. However, the most important result of the evaluation is that the proposed mechanism improves the fairness in the network capacity sharing. Considering the throughput experienced by client stations, our proposal provides to all clients equal average throughputs. Additionally, our mechanism also avoids performance degradation of legacy user communications.

The performance of the two presented virtualization techniques differs only in one aspect: the fairness index results. However, slight differences in the fairness index [15] results represent large differences in the throughput distribution experienced by the clients. Thus, as the main objective of the proposed mechanism is to provide better load balancing to the clients, the dynamic virtualization technique is the best choice when compared to the other mechanisms evaluated.

As future works we intend to compare the performance of the dynamic virtualization to other mechanisms in the literature. We also intend to develop a prototype of the proposed virtualization mechanism using commodity 802.11 network devices supported by the open source MadWifi driver. 


\section{References}

1. Y. Bejerano, S.-J. Han, and L. E. Li, "Fairness and load balancing in wireless LANs using association control," in MobiCom '04: Proceedings of the 10th annual international conference on Mobile computing and networking. New York, NY, USA: ACM, September 2004, pp. 315-329.

2. E. G. Villegas, R. V. Ferre, and J. P. Aspas, "Cooperative load balancing in IEEE 802.11 networks with cell breathing," Computers and Communications, 2008. ISCC 2008. IEEE Symposium on, pp. 1133-1140, July 2008.

3. S.-T. Sheu and C.-C. Wu, "Dynamic Load Balance Algorithm (DLBA) for IEEE 802.11 wireless LAN," Tamkang Journal of Science and Engineering, vol. 2, pp. 45-52, 1999.

4. S. Paul and S. Seshan, "Technical document on wireless virtualization," GENI: Global Environment for Network Innovations, Tech. Rep., September 2006.

5. P. Bahl, P. Bahl, and R. Chandra, "MultiNet: Connection to multiple IEEE 802.11 networks using a single wireless card," Microsoft Research, Redmond, WA, Tech. Rep., August 2003.

6. P. Barham, B. Dragovic, K. Fraser, S. Hand, T. Harris, A. Ho, R. Neugebauer, I. Pratt, and A. Warfield, "Xen and the art of virtualization," in SOSP '03: Proceedings of the nineteenth ACM symposium on Operating systems principles. New York, NY, USA: ACM, 2003, pp. 164-177.

7. A. Adya, P. Bahl, R. Chandra, and L. Qiu, "Architecture and techniques for diagnosing faults in IEEE 802.11 infrastructure networks," in MobiCom '04: Proceedings of the 10th annual international conference on Mobile computing and networking. New York, NY, USA: ACM, 2004, pp. 30-44.

8. W.-C. Wang, C.-H. Hsu, Y.-M. Chen, and T.-Y. Chung, "SCTP-based handover for VoIP over IEEE 802.11 WLAN using device virtualization," Advanced Communication Technology, The 9th International Conference on, vol. 2, pp. 1073-1076, February 2007.

9. I. Ramine and S. Savage, "SyncScan - Practical fast handoff for 802.11 infrastructure network," INFOCOM'05, vol. 1, pp. 675-684, 13-17 March 2005.

10. G. Smith, A. Chaturvedi, A. Mishra, and S. Banerjee, "Wireless virtualization on commodity 802.11 hardware," in WinTECH '0\%: Proceedings of the the second ACM international workshop on Wireless network testbeds, experimental evaluation and characterization. Montreal, Quebec, Canada: ACM, September 2007, pp. 75-82.

11. R. Mahindra, G. D. Bhanage, G. Hadjichristofi, I. Seskar, D. Raychaudhuri, and Y. Y. Zhang, "Space versus time separation for wireless virtualization on an indoor grid," Next Generation Internet Networks, 2008. NGI 2008, pp. 215-222, April 2008.

12. G. Athanasiou, T. Korakis, O. Ercetin, and L. Tassiulas, "Dynamic cross-layer association in 802.11-based mesh networks," INFOCOM 200\%. 26th IEEE International Conference on Computer Communications. IEEE, pp. 2090-2098, May 2007.

13. S. Bangolae, C. Bell, and E. Qi, "Performance study of fast BSS transition using IEEE 802.11r," in IWCMC '06: Proceedings of the 2006 international conference on Wireless communications and mobile computing. New York, NY, USA: ACM, 2006, pp. 737-742.

14. "Part 11: Wireless LAN Medium Access Control (MAC) and Physical Layer (PHY) specifications," IEEE Std. 802.11, August 1999.

15. R. K. Jain, D.-M. W. Chiu, and W. R. Hawe, "A Quantitative Measure of Fairness and Discrimination for Resource Allocation in Shared Computer System," Digital Equipament Corporation, Maynard, MA, USA, DEC Research Report TR-301, September 1984. 